\title{
Ensino de astronomia: uma abordagem didática a partir da Teoria da Relatividade Geral
}

\author{
Teaching Astronomy: a didactic approach based on the Theory of General Relativity \\ Marcello Ferreira $^{*} @$, Roberto Vinícios Lessa do Couto $^{1}$, Olavo Leopoldino da Silva Filho \\ Laura Paulucci ${ }^{2}$, Fábio Ferreira Monteiro ${ }^{1 \oplus}$ \\ ${ }^{1}$ Universidade de Brasília, Instituto de Física, Brasília, DF, Brasil. \\ ${ }^{2}$ Universidade Federal do ABC, Santo André, SP, Brasil.
}

\begin{abstract}
Recebido em 23 de abril de 2021. Revisado em 10 de setembro de 2021. Aceito em 23 de setembro de 2021.
A partir da análise de uma articulação entre as perspectivas cognitivistas da aprendizagem significativa de David Ausubel e educacionais das comunidades de investigação presentes no programa de filosofia de Matthew Lipman, este artigo discute o desenvolvimento de um produto educacional acerca de tópicos de astronomia. A aplicação foi feita em uma turma do terceiro ano do ensino médio da rede pública do Distrito Federal. As aulas foram distribuídas em quatro grandes temas: gravitação universal e sistema solar; relatividade geral; buracos negros; e lentes gravitacionais. Conteúdos, atividades e abordagens diversificados foram mediados por tecnologias educacionais digitais e as avaliações consideraram a proficiência como níveis de expressão das respectivas teorias psicológica e educacional. Os resultados revelaram o entusiasmo e interesse dos estudantes pelos temas propostos. Também foi possível verificar indicadores do desenvolvimento de habilidades de raciocínio, formação de conceitos, investigação, tradução e indícios de aprendizagem significativa. Por fim, foi possível concluir que a inserção de temas ligados à astronomia e à física moderna e contemporânea são viáveis no ensino médio, mesmo no contexto precário da escola pública, em contraposição ao currículo atual de física, desgastado e baseado principalmente na física clássica e em abordagens metodológicas muitas vezes difusas.
\end{abstract}

Palavras-chave: Ensino de física, Astronomia, Relatividade Geral, Aprendizagem Significativa, Comunidades de investigação.

From the analysis of an articulation between the cognitive perspectives of David Ausubel's meaningful learning and the educational ones of the research communities present in Matthew Lipman's philosophy program, this article discusses the development of an educational product on astronomy topics. The application was made in a third-year class of public high school in the Federal District. The classes were divided into four major themes: universal gravitation and the solar system; general relativity; black holes; and gravitational lenses. Diversified content, activities and approaches were mediated by digital educational technologies and the evaluations considered proficiency as levels of expression of the respective psychological and educational theories. The results revealed the students' enthusiasm and interest in the proposed themes. It was also possible to verify indicators of the development of reasoning skills, concept formation, investigation, translation, and evidence of significant learning. Finally, it was possible to conclude that the insertion of themes related to astronomy and modern and contemporary physics are viable in high school, even in the precarious context of the public school, in contrast to the current physics curriculum, worn out and based mainly on classical and methodological approaches that are often diffuse.

Keywords: Physics teaching, Astronomy, General relativity, Meaningful learning, Communities of inquiry.

\section{Introdução}

A astronomia, uma das ciências mais antigas da humanidade, faz parte da curiosidade do senso comum e está entre os temas que mais despertam o interesse dos jovens na faixa de 15 anos de idade, o que corresponde ao público que tipicamente inicia o ensino médio no Brasil [1, 2]. Por ser um tema popular, e reconhecidamente fundante do pensamento científico moderno, diversos países a inserem em seus currículos de ciências.

\footnotetext{
*Endereço de correspondência: marcellof@unb.br
}

No Brasil, os Parâmetros Curriculares Nacionais para o Ensino Médio - PCN+ EM [3] incluem a astronomia em seu sexto tema estruturador, denominado "Universo, Terra e Vida", e mencionam a importância de propiciar aos estudantes uma visão da estrutura e da organização do cosmos, permitindo que eles possam se situar no universo e compreender descobertas divulgadas pelos meios de comunicação, como, por exemplo, a imagem de um buraco negro, apresentada pela primeira vez em 2019. Os PCN+ EM destacam, ainda, como parte do currículo, a interação gravitacional e os métodos de investigação de estrelas e galáxias, como a lei de 
gravitação universal, a relatividade geral e as lentes gravitacionais.

Em outro documento oficial, a Base Nacional Comum Curricular 4] 1] o tema está inserido na segunda competência específica para o ensino médio, que os estudantes devem desenvolver para a área de ciências da natureza e suas tecnologias. Destaca que o estudante deve ser capaz de construir e utilizar interpretações acerca da dinâmica da Vida, da Terra e do Cosmos para elaborar argumentos, realizar previsões acerca do funcionamento e da evolução dos seres vivos e do universo, bem como fundamentar e defender decisões éticas e responsáveis.

Portanto, é oportuno apresentar a evolução de modelos científicos, como a gravitação de Newton e a Relatividade de Einstein, pois, além dos tópicos constarem dos documentos curriculares oficiais, também implicam uma visão da ciência como processo em constante evolução, usualmente apartada das preocupações no ensino médio. A astronomia, por outro lado, apresenta potencial interdisciplinar e possibilidades para sustentar discussões socioeconômicas e culturais [5], podendo ser relacionada a vários campos do saber.

Contudo, mesmo em face a tantas potencialidades temáticas e metodológicas, os estudantes são usualmente pouco expostos aos conhecimentos acerca da astronomia, dentro ou fora da sala de aula. Isso se dá, por um lado, pelas limitações de um sistema de ensino que curricularizou as ciências de modo tardio e desqualificado e, por outro, pelos drásticos passivos educacionais que comprometem e desfavorecem a ciência e a discussão acerca da sua natureza como atributos de uma formação escolar plena. Dentre os diversos motivos para isso, podese citar a precariedade da qualificação dos professores para lidar com esse assunto; professores que ocupam vagas fora de sua área de formação; difusão, tanto por professores quanto por estudantes, de concepções presentes no senso comum e sem concordância com o conhecimento produzido pela comunidade científica; e a baixa qualidade dos livros didáticos disponibilizados, que, comumente, dedicam pouca ênfase, quando o fazem, à astronomia, lidando com seus conteúdos de forma superficial e incompleta e, até mesmo, com recurso a sistemáticos erros históricos e conceituais [6] 2

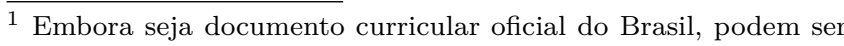
tecidas críticas contundentes com relação à BNCC. Consideramos, por exemplo, que ela é resultado de frágil articulação política e de pouco diálogo institucional, além de objeto de enfraquecimento da autonomia curricular das redes de ensino e dos professores, mesmo que tenha a regionalização como um de seus princípios. Por fim, sob o argumento de homogeneizar a diversidade escolar brasileira, ela, de fato, corrobora compromissos editoriais e econômicos e a lógica hierarquizante de resultados em exames de aferição de conhecimentos em âmbito nacional e internacional, como o Enem e o Pisa, por exemplo.

2 O desinteresse editorial pela atualização de temas científicos e pela abordagem de física moderna e contemporânea e de astronomia mereceria uma discussão à parte. Não havendo aqui ocasião para fazê-la, detemo-nos a constatar essa limitação e registrar
}

Em fevereiro de 2020, pesquisa realizada na base de dados da Scielo e no catálogo de teses e dissertações da Capes evidenciou que, entre 2014 e 2020, foram apresentadas, no âmbito de programas de pós-graduação stricto sensu em ensino de física (e, em particular, no MNPEF - Mestrado Nacional Profissional em Ensino de Física), número pouco expressivo de dissertações e produtos educacionais associados ao tema Relatividade Geral. A seguir, sem pretensão de esgotar a literatura disponível e com finalidades singularmente ilustrativas, apresentamos e sintetizamos quatro (4) desses trabalhos, priorizando abordagens mais recentes.

Sá [7, em 2015, fez uma proposta de intervenção didática, a partir da Transposição Didática de Yves Chevallard, contendo planos de aula e materiais de apoio acerca dos seguintes temas: história da mecânica, $2^{\mathrm{o}}$ postulado da relatividade restrita, dilatação do tempo e contração do espaço, deformação do espaço-tempo, limitações da mecânica newtoniana, equivalência massa/energia e desvio da trajetória da luz. Ele relata que seu produto educacional foi capaz de motivar os estudantes e de promover uma visão histórico-coletiva da ciência e afirma que sua sequência didática foi capaz de promover níveis "de aprendizado dos conceitos relativísticos igual ou superior aos dos conceitos da Mecânica Clássica tradicionalmente ensinada".

Também em 2015, Freire 8 fez uso da Teoria da Aprendizagem Significativa (TAS) de David Ausubel para, a partir de material didático alternativo no estilo mangá, ensinar tópicos de relatividade restrita e geral, como o princípio da equivalência, a curvatura do espaço-tempo e os buracos negros. Ele relata que a sequência didática foi bem recebida pelos estudantes, que demonstraram apreço pelo tema abordado, e que, ao final, identificou "uma parcela considerável dentre eles que assimilou o novo conhecimento, modificando subsunçores evidenciados nas atividades iniciais".

Em 2017, Souza 9] também utilizou a TAS para, por meio de hipermídias, ensinar conteúdos relativos à gravitação universal, ao princípio da equivalência, à precessão de Mercúrio e à cosmologia de forma superficial, ao abordar o paradoxo de Olbers, a geometria do universo, a radiação cósmica de fundo, a matéria escura e a energia escura em uma única aula. Ele relata que os estudantes se envolveram e se comprometeram com as atividades propostas, demonstrando pontualidade, assiduidade e interesse pelos temas apresentados, bem como afirmaram que a hipermídia favoreceu suas aprendizagens.

Novamente em 2017, Oliveira [10] fez uso do referencial da TAS de David Ausubel e construiu material interativo, utilizando ambientes virtuais de aprendizagem para ensinar a gravitação universal e os buracos

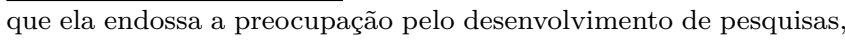
aplicações educacionais e materiais instrucionais complementares nesses temas. 
negros. Além disso, apresentou em seu material de ensino uma seção dedicada aos fundamentos teóricos dos conteúdos abordados. Ele relatou que o interesse dos estudantes pelo tema cresceu à medida que as atividades eram propostas, culminando na criação de um clube de astronomia, bem como que houve aumento nos desempenhos escolares.

Nesses trabalhos, foram produzidas propostas de intervenções didáticas envolvendo frações da teoria da relatividade, o que, à primeira vista, pode ter a faculdade de introdução do tema, mas que, detidamente analisado, poderia induzir visões compartimentadas do tema. Isso fica mais evidente quando se nota que somente em Oliveira [10] foi apresentado conteúdo teórico voltado exclusivamente ao professor, o que pretensamente contribui para ampliar o conhecimento acerca do assunto e a segurança didática.

Em face das lacunas de abordagem evidenciadas, e buscando uma produtividade alternativa, investigamos a possibilidade da articulação entre a Teoria da Aprendizagem Significativa de David Ausubel e o Programa de Filosofia para Crianças e Adolescentes (PFCA) de Matthew Lipman [5. Como resultado, foi organizado um produto educaciona 3 com o compromisso de apresentar estratégias didáticas voltadas à astronomia no ensino médio, em abordagem relativamente simplificada, porém não fragmentada, a partir da teoria da relatividade geral e, ao mesmo tempo, apresentar também conteúdo teórico voltado ao professor, de modo a incentivar a aplicação da proposta.

O objetivo deste artigo, portanto, é apresentar uma análise da articulação teórico-metodológica desenvolvida na elaboração das estratégias didáticas utilizadas no referido produto educacional e avaliar, a partir das respostas dos estudantes, indicadores de desenvolvimento de habilidades de raciocínio, formação de conceitos, investigação, tradução e indícios de aprendizagem significativa. Isso se dará pela articulação de um referencial teórico eminentemente descritivo, de matriz cognitivista, que é a aprendizagem significativa, a outro de perspectiva normativa, que é a filosofia para crianças e adolescentes, cuja produtividade foi avaliada e mostrada evidente em outros trabalhos [11-13].

\section{Fundamentação Teórica}

No campo da aprendizagem, enquanto as teorias psicológicas, de natureza eminentemente descritiva, se dedicam aos fenômenos relacionados aos processos de aquisição, retenção e utilização do conhecimento para compreender as condições necessárias para a ocorrência da aprendizagem, as teorias educacionais, de natureza

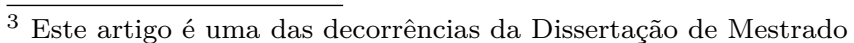
intitulada "Astronomia no Ensino Médio: uma abordagem simplificada a partir da Relatividade Geral" [5, defendida em 2020 no MNPEF da UnB.
}

eminentemente normativa, se dedicam aos procedimentos para que se alcance a aprendizagem, levando-se em conta o tipo de indivíduo que se deseja formar, em determinadas condições. Neste último, os métodos e valores enfatizam ainda aspectos culturais, sociais, éticos, morais dentre outros [12 14].

As teorias psicológicas e educacionais são, de fato, complementares e estruturam de maneira mais complexa o processo de ensino-aprendizagem. Nesse sentido, uma articulação entre as ideias descritivas de David Ausubel com as ideias normativas de Matthew Lipman permite, de um lado, apresentar aportes teóricos relativos ao processo da assimilação do conhecimento a partir de outros conhecimentos já presentes na estrutura cognitiva dos estudantes, os subsunçores, e, do outro lado, enfatizar o pensamento de ordem superior, visando desenvolver nos estudantes a capacidade crítica a respeito da sociedade em que estão inseridos. Tomamos tal estrutura como o arranjo hierarquizado e sequencial de fatos, conceitos, proposições, teorias e conhecimento bruto que um indivíduo tem à sua disposição, internamente, em determinado momento.

Assim, enquanto Ausubel descreve os mecanismos segundo os quais um conhecimento pode ser assimilado na estrutura congnitiva, relacionando-se de forma hierarquizada com seus respectivos subsunçores, Lipman prescreve um método, as comunidades de investigação, capaz de auxiliar os aprendizes a desenvolverem suas habilidades para alcançarem o pensar de ordem superior. Somente este último, concretamente, aborda o fenômeno cognitivo em perspectiva social (no limite, escolar).

É possível, portanto, perceber que a aplicação apenas da teoria de Ausubel, que é descritiva, se mostraria insuficiente - ou incongruente, a rigor - em um contexto concreto de sala de aula, uma vez que ela não considera aspectos específicos dos indivíduos participantes do processo educacional. Em outros termos, apesar de possuir elementos relativos à aquisição de conhecimento essencialmente abstratos, essa teoria pouco diz (porque não devotada a) acerca de como ensinar determinado estudante em contexto escolar típico. Com isso, a articulação com a proposta de Lipman, que é normativa, adiciona ao processo as variáveis não previstas, relativas aos indivíduos e à forma de ensiná-los.

O amálgama entre esses dois referenciais teóricos pode ser encontrado em Silva Filho et al. [12] e em Silva Filho e Ferreira [13].

\section{Funções Pedagógicas e Estratégias Didáticas}

$\mathrm{Na}$ aplicação da sequência didática ${ }^{4}$ a perspectiva da TAS 13 15, forneceu os subsídios cognitivos relacionados a como ocorre o processo de assimilação de conhecimento que resulta na aprendizagem significativa,

\footnotetext{
${ }^{4}$ Conferir com o produto educacional integrante de 5 .
} 
enquanto a perspectiva de Lipman, por meio de seu PFCA, proveu elementos teórico-metodológicos (normativos) necessário ao seu alcance.

Desse modo, os temas de estudo foram hierarquizados didaticamente considerando o interesse dos estudantes pela astronomia e seus conhecimentos prévios, mesmo que escassos, para formalizar uma intervenção com potencial de produzir uma aprendizagem significativa. Também foi levada em consideração a figura do estudante e o contexto no qual ele está inserido, para apresentar metodologia de ensino capaz de identificar em que nível a aprendizagem ocorreria.

Os tópicos de astronomia, com abordagem simplificada a partir da teoria da relatividade geral, foram organizados em 20 aulas duplas, ou 10 encontros, distribuídos em 4 grandes módulos de ensino: M1 - gravitação universal e sistema solar; M2 - relatividade; M3 buracos negros; e M4 - lentes gravitacionais. As aulas de cada módulo foram organizadas em estratégias didáticas, considerando cinco funções pedagógicas fundamentais:

a. INVESTIGAÇÃO: Formação de pequenos grupos ou comunidades de investigação, em que os estudantes possam se organizar, investigar e discutir as diversas atividades apresentadas: figuras, vídeos, simulações, textos de apoio, questionários e questionamentos.

b. ORGANIZAÇÃO PRÉVIA: Apresentação de imagens, vídeos, experiências, dinâmicas ou simulações acerca do tema em estudo acompanhados de questionamentos acerca do que os estudantes veem, percebem ou entendem o que é apresentado.

c. DIFERENCIAÇÃO PROGRESSIVA: Leitura de textos de apoio, mediação do professor e explicações do professor acerca de conceitos e formulações matemáticas relacionados ao tema em estudo.

d. RECONCILIAÇÃO INTEGRATIVA: Explicações do professor que resumem e estabelecem relações de significado entre as imagens, vídeos, experiências e simulações com os conceitos e as formulações matemáticas do tema em estudo.

e. VERIFICAÇÃO DE APRENDIZAGEM: Questionamentos, questionários e observação da participação dos estudantes nas discussões e nas dinâmicas para fazer inferência acerca dos subsunçores dos estudantes.

\section{Metodologia}

A metodologia desenvolvida neste trabalho é do tipo translacional [5, 11], isto é, se dá sob a integração de processos educativos, produtos e tecnologias sociais por profissional em serviço, com mediação de conhecimento acadêmico e retroalimentação, buscando analisar a interconexão dos dois referenciais teóricos já mencionados e sua aplicação em contexto típico de ensino.
Desse modo, o processo de análise dos efeitos das estratégias didáticas ocorre em todos os tempos e cada uma das funções pedagógicas utilizadas nas aulas podem ter um nível de avaliação específico:

a. na INVESTIGAÇÃO, a qualidade da interação entre os estudantes no sentido de entender melhor os conceitos, uma imagem, uma experiência, uma simulação ou um fenômeno apresentado poderá indicar o bom ou mau funcionamento das comunidades de investigação.

b. na ORGANIZAÇÃO PRÉVIA, a qualidade das respostas dos estudantes aos questionamentos acerca de imagens, vídeos, experiências, dinâmicas ou simulações acerca do tema em estudo poderá indicar a presença ou ausência de subsunçores na estrutura cognitiva dos estudantes.

c. na DIFERENCIAÇÃO PROGRESSIVA, a qualidade da interação dos estudantes com perguntas e comentários ao professor, ou colegas, relativos aos textos de apoio e as explicações ou mediação do professor poderá indicar a ocorrência de diferenciação progressiva dos subsunçores, e o interesse dos estudantes pelos conceitos em estudo.

d. na RECONCILIAÇÃO INTEGRATIVA a qualidade das Interrupções dos estudantes relativas às relações de significado entre as imagens, vídeos, experiências e simulações com os conceitos e as formulações matemáticas do tema em estudo poderá indicar a ocorrência reconciliação integrativa do subsunçores.

e. na VERIFICAÇÃO DE APRENDIZAGEM a observação das respostas aos questionamentos para fazer inferência acerca dos subsunçores dos estudantes; observação das interrupções dos estudantes com perguntas e comentários para fazer inferência acerca da ocorrência de diferenciação progressiva dos subsunçores; e, observação de respostas dadas aos questionamentos, aos itens dos questionários, e dos comentários dos estudantes, que abordem os temas em estudo de modo diferente do apresentado nos textos de apoio ou nas explicações do professor, para avaliar indícios de aprendizagem significativa.

De modo geral, cada função pedagógica apresentou resultado satisfatório durante a aplicação do produto educacional e os estudantes se envolverem com entusiasmo e interesse pelos temas propostos. Também foi possível identificar, em cada módulo de ensino, o desenvolvimento das habilidades de raciocínio, formação de conceitos, investigação, tradução e de indícios de aprendizagem significativa, como detalhado a seguir.

\section{Análise das Estratégias Didáticas}

O produto educacional foi aplicado em uma turma do terceiro ano do ensino médio de uma escola pública 
localizada em Ceilândia, região administrativa do Distrito Federal, em novembro do ano de 2019. A turma, com 29 estudantes, possibilitou a auto-organização de 8 grupos (como comunidades de investigação), uma vez que a afinidade entre seus membros é um facilitador do diálogo almejado pela proposta.

Uma característica dos estudantes da escola é o alto índice de ausências às aulas e a turma de aplicação não fugiu a esse padrão, apresentando média de $31 \%$ de estudantes faltantes por aula. As ausências não se concentravam nos mesmos estudantes, e eram distribuídas de forma quase homogênea por toda a turma, o que afetou negativamente seu desempenho de modo geral. Os trabalhos de Pontili e Kassouf [17] e de Costa, Guimarães e Rocha 18 listam alguns fatores que estimulam a baixa frequência escolar, como a renda per capita da família dos estudantes, a escolaridade do chefe da família, a cor dos estudantes (negros e pardos têm maior probabilidade de faltarem às aulas que brancos), problemas econômicos e familiares, desmotivação e aspectos físicos e organizacionais da escola.

O público da escola onde foi aplicado o produto educacional se enquadra no rol citado, pois é composto, principalmente, por moradores do Pôr do Sol e do Sol Nascente, regiões com baixa escolaridade: $64,15 \%$ não possuem o ensino médio completo; baixa renda per capita: $\mathrm{R} \$ 622,30$; e de maioria negra ou parda: $66,83 \%$ se declaram dessa forma [19.

Embora, a partir desses dados, seja possível inferir o porquê do alto número de ausências dos estudantes, apenas uma pesquisa regional e específica poderia investigar correlações estatisticamente apropriadas. Seguramente, há outras interveniências à evasão escolar, relacionados à personalidade e às condições pedagógicas particulares, mas que, por escopo, não serão desenvolvidas nesta discussão. Importa ressaltar, entretanto, que o número de ausências observado na pesquisa teve impactos na aprendizagem dos estudantes, uma vez que dele decorria um contato intermitente, irregular e descontínuo com o conteúdo, o que, em geral, dificulta a aquisição ou a modificação dos subsunçores existentes em suas estruturas cognitivas. Desse modo, a própria aprendizagem significativa é prejudicada, pois é altamente dependente dos conhecimentos prévios dos estudantes.

Nos dedicamos a seguir, enfaticamente, à análise dos resultados da aplicação didática na referida perspectiva translacional.

\subsection{Gravitação Universal e Sistema Solar}

\subsubsection{Estratégias didáticas}

a. Víded ${ }^{5}$ de uma adaptação do experimento de Cavendish.

\footnotetext{
5 O vídeo faz uma demonstração qualitativa do experimento de Cavendish, demonstrando a atração gravitacional entre corpos, e se encontra na plataforma Youtube. Disponível em https://www. youtube.com/watch?v=11sLusnVZwM. Acesso em: 12 abr. 2021.
}

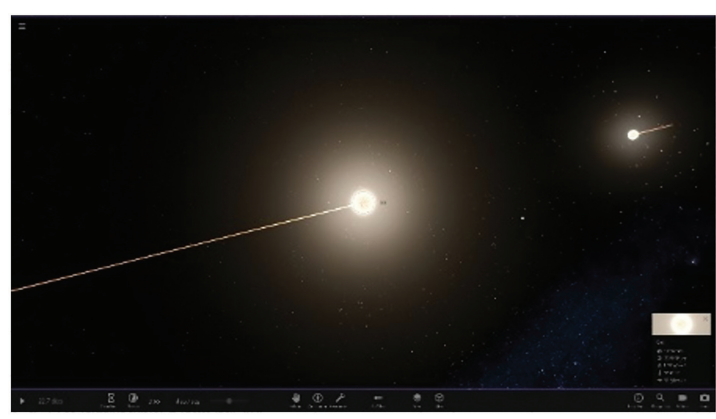

Figura 1: Simulação da colisão de duas estrelas. Fonte: Universe Sandbox (2015) [20].

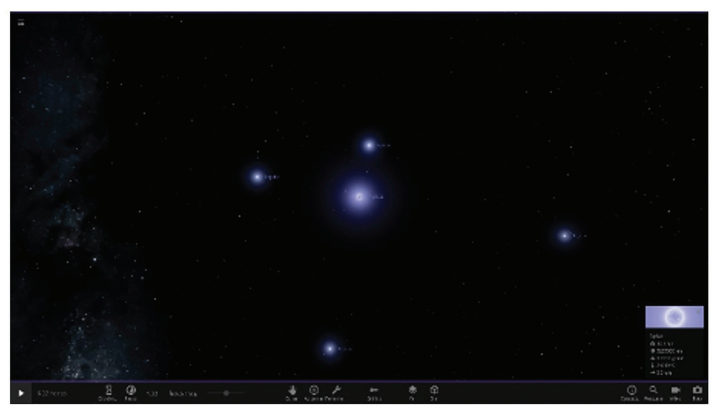

Figura 2: Simulação da colisão de 4 estrelas com uma estrela central.

Fonte: Universe Sandbox (2015) [21].

b. Experimento de queda livre, mediado pelo abandono de objetos a determinada altura e investigação, entre os alunos, acerca da relação entre esse fenômeno e aquele apresentado no vídeo.

c. Explicações acerca da atração entre as massas e força gravitacional.

d. Explicações acerca da universalidade da força gravitacional.

e. Simulações ${ }^{6}$ computacionais acerca de colisões de duas estrelas (massas iguais e diferentes) (Figura 1).

f. Formação de pequenos grupos de investigação para trabalhar no "roteiro de discussão 1.1".

g. Explicações acerca da proporcionalidade entre as massas que se atraem, a força gravitacional e a aceleração do movimento.

h. Solicitação aos grupos de investigação para discutirem o item 1 do "questionário 1.1".

i. Simulações computacionais entre uma estrela muito massiva ao centro e quatro estrelas menores com massas iguais, mas a distâncias diferentes (Figura 2).

j. Apresentação, por simulação, de que, com exceção do momento inicial, as velocidades das estrelas são diferentes em cada instante.

6 O simulador se encontra disponível na plataforma de jogos eletrônicos Steam. Disponível em: https://store.steampowered .com/app/230290/ Acesso em: 12 abr. 2021. 
k. Interação entre os grupos de investigação baseada no "roteiro de discussão 1.2".

1. Explicações acerca da relação entre a força de atração gravitacional e a distância entre as massas.

m. Explicações acerca da não linearidade da relação entre a força de tração gravitacional e a distância entre as massas.

n. Solicitação para que os grupos de investigação discutam o item 2 do "questionário 1.1".

o. Explicações acerca da proporção $F \propto \frac{m_{1} m_{2}}{r^{2}}$, em que $m_{1}$ e $m_{2}$ são as massas dos corpos e $r$ é a distância entre eles, e introdução da constante gravitacional $G=6,67 \cdot 10^{-11} \frac{\mathrm{Nm}^{2}}{\mathrm{~kg}^{2}}$, discutindo que a força gravitacional é dada por $F=G \frac{m_{1} m_{2}}{r^{2}}$.

p. Solicitação para que os grupos de investigação discutam o item 3 do "questionário 1.1".

q. Explicações acerca da relação entre as órbitas dos planetas e a força gravitacional entre o Sol e os planetas.

r. Explicações acerca da relação entre a força gravitacional a forma esférica dos planetas e do Sol.

s. Simulações computacionais acerca do Sistema Solar, mostrando seus principais componentes (o Sol, os planetas e suas órbitas).

t. Solicitação para que os grupos de investigação leiam e discutam o "texto de apoio 1.1".

u. Solicitação para que os grupos de investigação discutam os itens do "questionário 1.2".

\subsubsection{Questionários e roteiros}

- Questionário 1.1 - Gravitação Universal 1) Por que o tempo de colisão das estrelas é diferente em cada simulação? 2) Por que a velocidade das estrelas em cada instante específico é diferente, mesmo elas sendo todas iguais? 3) Por que não se observa, no cotidiano, dois corpos quaisquer se aproximando devido a força gravitacional?

- Questionário 1.2 - Gravitação e Sistema Solar 1) Se a força gravitacional do Sol faz com que todos os corpos sejam atraídos para ele, por que os planetas não caem no Sol? 2) O que aconteceria com a órbita dos planetas do Sistema Solar se o Sol desaparecesse subitamente? 3) Por que os quatro planetas mais distantes do Sol possuem mais satélites que os demais?

- Roteiro de discussão $\mathbf{1 . 1}$ 1) Quais são as principais diferenças entre cada simulação? 2) $\mathrm{O}$ que aconteceria caso fossem realizadas outras simulações com estrelas ainda mais massivas? 3) Qual é a relação entre a massa das estrelas e o tempo de colisão? 4) Qual é a relação entre a massa das estrelas e a força gravitacional?

- Roteiro de discussão 1.2 1) Quais são as principais diferenças entre cada estrela da simulação? 2) O que aconteceria caso fossem realizadas outras simulações com as estrelas posicionadas mais distantes da estrela central? 3) Qual é a relação entre a distância das estrelas e suas respectivas velocidades instantâneas? 4) Qual é a relação entre a distância das estrelas e a força gravitacional?

\subsubsection{Análise das estratégias didáticas}

Nas fases de organização prévia, estratégias "a" e "b", os estudantes demonstraram surpresa ao verem pesos presos à régua serem atraídos pelas bolas de boliche, indicando ausência de subsunçores acerca da gravitação.

Nas fases de investigação, estratégias "f" e "k", os estudantes revelaram certo nível de compreensão do fenômeno em estudo, mas também a ausência de alguns subsunçores relacionados à força gravitacional.

Nas fases de diferenciação progressiva, estratégias "e", "g", "i", "j", "l", "m", "q", "q", "r", "s" e "t", os estudantes revelaram interesse pelo assunto e pelas estratégias utilizadas. Durante as simulações, eles se mostraram atentos e conseguiram relacionar os dados apresentados aos fenômenos simulados, indicando uso de habilidades de ordem superior de raciocínio, formação de conceitos, investigação e tradução.

Nas fases de reconciliação integrativa, estratégias "c", "d" e "o", as explicações, principalmente acerca a expressão matemática da lei de gravitação universal, houve pouca participação dos estudantes, possivelmente pela ausência de subsunçores relacionados à modelagem de expressões matemáticas e análise de funções.

Nas fases de verificação de aprendizagem, estratégias "h", "n", "p" e "u", as respostas dos estudantes revelaram certo nível de compreensão do tema em estudo, mas também a ausência de alguns subsunçores relacionados a vetores, funções e à força gravitacional.

\subsection{Relatividade}

\subsubsection{Estratégias didáticas}

a. Formação de pequenos grupos de investigação para discutir o "texto de apoio 2.1".

b. Mediação da discussão entre os grupos de investigação com base no "roteiro de discussão 2.1 ".

c. Solicitação, aos grupos de investigação, para que discutam os itens do "questionário 2.1".

d. Realização, com os estudantes, da dinâmica do espaço-tempo.

e. Apresentação de cenas do filme ${ }^{7}$ Interestelar.

f. Mediação da discussão, nos grupos de investigação, com base no "roteiro de discussão $2.2 "$.

g. Explicações acerca da natureza do espaço e do tempo.

h. Explicações acerca do quadrivetor espaço-tempo.

\footnotetext{
7 Interestelar. Direção de Christopher Nolan. EUA: Warner Bros. Pictures, 2014. 1 disco blu-ray (169 min).
} 
i. Explicações acerca da constância da velocidade da luz e sua consequência para o espaço e o tempo.

j. Explicações acerca de como espaço e o tempo podem ser alterados como consequência do movimento ou da gravidade.

k. Explicações acerca da relatividade do espaço e do tempo e suas relações com a escolha do referencial.

1. Apresentação de um gif animado acerca da distorção do espaço-tempo.

m. Realização de experimento do lençol.

n. Formação de pequenos grupos de investigação, para discutir os itens do "questionário 2.2".

o. Questionamentos aos estudantes acerca da possibilidade da aceleração e da gravidade serem equivalentes.

p. Explicação do experimento mental do elevador proposto por Einstein para mostrar a equivalência entre aceleração e gravidade.

q. Solicitação, aos grupos de investigação, para que discutam possíveis consequências dessa equivalência.

r. Solicitação, aos grupos de investigação, para que discutam os itens do "questionário 2.3".

\subsubsection{Questionários e roteiros}

- Questionário 2.1 - Espaço e Tempo 1) Tendo como base uma visão realista da física, discorra acerca da existência do espaço e do tempo. 2) Como é obtida a medida da grandeza física distância? 3) Explique o conceito de espaço. 4) Explique o conceito de tempo. 5) Tendo como base a forma como são obtidas as medidas de tempo, é possível obter um valor exato para o tempo? Explique sua resposta.

- Roteiro de discussão 2.1 1) O que é o espaço? 2) O que é o tempo? 3) Eles existem realmente ou são invenções humanas? 4) O que é a medida de algo? 5) Como aumentar a precisão de uma medida?

- Roteiro de discussão 2.2 1) Ao voltar para a nave, o que eles perceberam que aconteceu com seu colega que ficou? 2) Quanto tempo o astronauta disse que passou para ele? 3) O tempo passou de forma diferente para eles? Isso é possível? 4) É possível saber quanto tempo os astronautas ficaram no planeta Miller? 5) Se o tempo pode passar de forma diferente para duas pessoas, vocês acham que o espaço é do mesmo tamanho em qualquer lugar? Vocês acham que o tamanho de um objeto pode mudar? 6) O que causou o efeito temporal sobre os astronautas? 7) Pode haver outra causa? 8) O que poderia causar a contração do espaço?

- Roteiro de discussão 2.3 1) Questionar os estudantes se o espaço e o tempo são grandezas distintas. As respostas devem ser na direção de que são diferentes. 2) Pedir que algum estudante se levante, vá para a porta da sala e verifique o horário em seu relógio. 3) Pedir que outro estudante se levante, ocupe o lugar que o primeiro estava e verifique o horário no mesmo relógio utilizado antes. 4) Pedir que os estudantes voltem aos seus lugares e questionar a turma qual é a posição dos dois estudantes no momento da medida no relógio. As respostas devem indicar que eles estavam no mesmo lugar. 5) Questionar a turma qual informação falta para entender por completo a posição dos estudantes. As respostas devem indicar que falta informar o horário em que a posição foi medida. 6) Pedir que dois estudantes se levantem. Um deles deve ocupar um canto da sala e o segundo deve ocupar outro canto da sala. Verificar a hora que o relógio marca. 7) Pedir que os estudantes voltem para seus lugares e perguntar se a medida do relógio é suficiente para entender por completo a posição dos estudantes.

- Dinâmica do Espaço-Tempo 1) Questionar os estudantes se o espaço e o tempo são grandezas distintas. As respostas devem ser na direção de que são diferentes. 2) Pedir que algum estudante se levante, vá para a porta da sala e verifique o horário em seu relógio. 3) Pedir que outro estudante se levante, ocupe o lugar que o primeiro estava e verifique o horário no mesmo relógio utilizado antes. 4) Pedir que os estudantes voltem aos seus lugares e questionar a turma qual é a posição dos dois estudantes no momento da medida no relógio. As respostas devem indicar que eles estavam no mesmo lugar. 5) Questionar a turma qual informação falta para entender por completo a posição dos estudantes. As respostas devem indicar que falta informar o horário em que a posição foi medida. 6) Pedir que dois estudantes se levantem. Um deles deve ocupar um canto da sala e o segundo deve ocupar outro canto da sala. Verificar a hora que o relógio marca. 7) Pedir que os estudantes voltem para seus lugares e perguntar se a medida do relógio é suficiente para entender por completo a posição dos estudantes. As respostas devem indicar que falta o local que cada aluno ocupava.

- Experimento do lençol Materiais: Um lençol tamanho solteiro, duas esferas pesadas (aproximadamente $5 \mathrm{~kg}$ cada), esferas leves (bolinhas de gude, de ping-pong, de tênis); Procedimentos a) Pedir que quatro estudantes segurem e estiquem o lençol, tentando manter a tensão constante. b) Colocar uma das esferas pesadas no centro do lençol esticado. c) Explicar que o lençol funciona como se fosse o espaço-tempo e, ao colocar a esfera no centro, ele sofreu uma alteração em seu formato, assim como o próprio espaço-tempo sofre uma alteração nas proximidades de corpos massivos. 
d) Complementar a explicação de que os modelos são apenas uma representação da realidade. Para isso, pode-se comparar o lençol e o modelo em 3D das figuras explicando que eles representam partes do espaço-tempo, mas não conseguem simular toda a natureza. O lençol, por exemplo, tem apenas duas dimensões, enquanto as figuras conseguem mostrar três dimensões, porém o espaço-tempo é uma entidade física com quatro dimensões. e) Jogar uma esfera leve no lençol esticado de modo a fazê-la dar uma volta na esfera pesada. f) Fazer uma comparação entre a volta dada pela esfera leve e a órbita de um planeta e explicar que a esfera leve não entra em órbita ao redor da pesada devido ao atrito com o lençol e com o ar, que a faz parar. g) Jogar várias esferas leves seguidamente com velocidades distintas de modo a tentar fazer algumas darem voltas em torno da esfera pesada e outras passem direto pelo lençol sem dar nenhuma volta. h) Explicar que as esferas mais lentas caem rapidamente para o centro do lençol, enquanto as mais rápidas passam direto por ele, sofrendo pequenas alterações no seu curso. Já as esferas com velocidades intermediárias conseguem dar uma ou duas voltas antes de caírem no centro. Porém, independente da velocidade, elas sempre sofrem algum desvio ao se aproximarem da esfera pesada, maior se passam próximas e menor se passam distantes. i) Recolher o material e pedir que os estudantes voltem para seus grupos.

\subsubsection{Análise das estratégias didáticas}

Nas fases de organização prévia, estratégias "d", "e" e "l", os estudantes demonstraram entusiasmo e interesse, mas também demonstraram espanto com as ondas gigantes e com o envelhecimento dos personagens mostrados nas cenas. Durante a discussão alguns estudantes fizeram inferências que extrapolaram as informações apresentadas, revelando indícios de aprendizagem significativa acerca do tema espaço-tempo, bem como o uso de habilidades de ordem superior de raciocínio.

Nas fases de investigação, estratégias "a", "b", "f", "q" e "r", os estudantes participaram ativamente e foi possível observar indícios de aprendizagem significativa acerca do tema espaço-tempo e algumas respostas revelaram a utilização de habilidades de ordem superior de raciocínio, formação de conceitos, investigação e tradução.

Nas fases de diferenciação progressiva, estratégias "h", "i", "j" e "k", os estudantes ficaram atentos, mas pouco participativos e evidenciaram uma aprendizagem apenas mecânica da ideia do universo em quatro dimensões.

Nas fases de avaliação de aprendizagem, estratégias "c" e "n", os estudantes apresentaram indícios de aprendizagem significativa acerca do tema espaço-tempo, bem como o uso de habilidades de ordem superior de raciocínio.
Nas fases de reconciliação integrativa, estratégias "m", "o" e "p", os estudantes demonstraram entusiasmo e interesse pelo tema. Além disso, a qualidade da participação com comentários e perguntas que extrapolaram os resultados do experimento, revelaram indícios de aprendizagem significativa, utilização de habilidades de raciocínio superior e de criatividade.

\subsection{Buracos Negros}

\subsubsection{Estratégias didáticas}

a. Apresentação de figuras acerca de buracos negros (Figura 3).

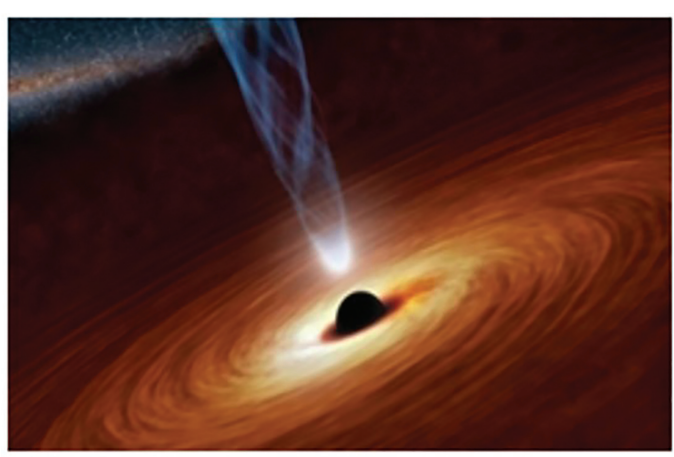

(a) Buraco negro Fonte: Nasa, 2013 21.

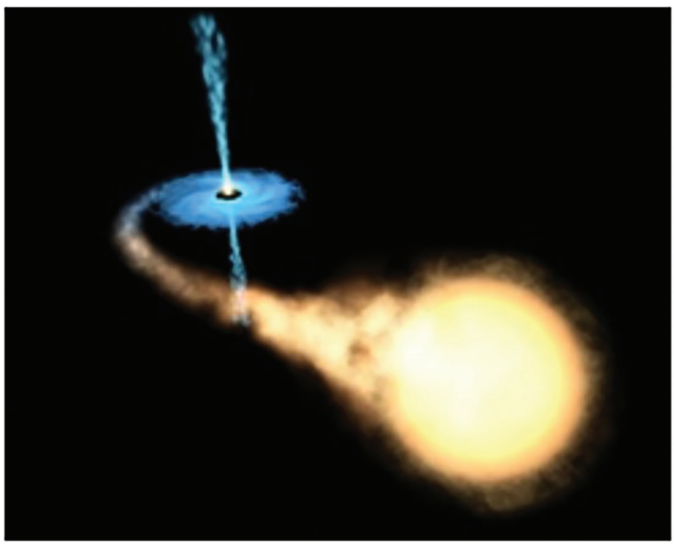

(b) Buraco negro sugando uma estrela. Fonte: Nasa, 2002 [22].

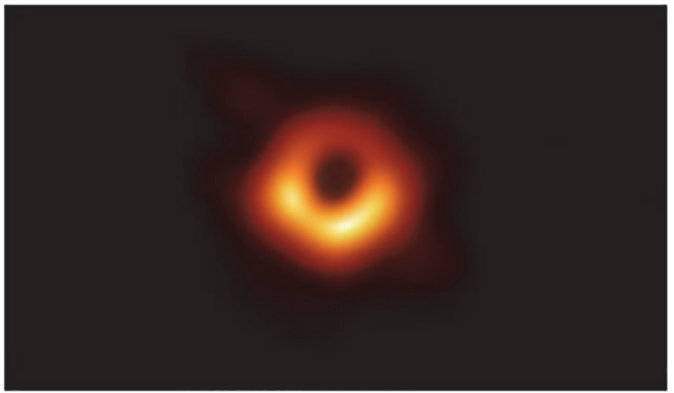

(c) Buraco negro e seu horizonte de eventos localizado na galáxia M87. Fonte: EHT, 2019 [23].

Figura 3: Concepções artísticas (a) e (b) e imagem (c) de um buraco negro. 
b. Questionamentos, aos estudantes, acerca do que tais figuras representariam (aspectos identificados, possíveis hipóteses para a degradação etc.).

c. Explicações acerca dos buracos negros e de suas relações com a massa e a gravidade.

d. Formação de pequenos grupos de investigação para discutir o "texto de apoio 3.1".

e. Mediação da discussão, entre os grupos de investigação, com base no "roteiro de discussão 3.1".

f. Solicitação aos grupos de investigação que discutam os itens do "questionário 3.1".

g. Explicações acerca do raio de Schwarzschild $r_{s}=\frac{2 G M}{c^{2}}$.

h. Apresentação do víded ${ }^{8}$ "Black Hole Size Comparison 2018".

i. Explicações acerca do horizonte de eventos de um buraco negro.

j. Explicações acerca da relação entre a massa e o volume no buraco negro.

k. Solicitação, aos grupos de investigação, para que discutam o "questionário 3.2".

1. Apresentação do víded" "Buracos Negros Estrelares".

m. Simulação do sistema solar em que o sol teria seu raio reduzido até o limite de Schwarzschild.

n. Solicitação, aos grupos de investigação, para que discutam o "texto de apoio 3.2".

o. Mediação da discussão entre os grupos de investigação, com base no "roteiro de discussão $3.2 "$.

p. Solicitação, aos grupos de investigação, para que discutam o "questionário 3.3".

q. Solicitação, aos grupos de investigação, para que discutam o "texto de apoio 3.3".

r. Explicações acerca da impossibilidade de obter uma imagem direta de um buraco negro.

s. Explicações acerca do fato de, somente em 2019, ter sido possível obter a primeira imagem do buraco negro.

t. Apresentação de reportagem ("Primeira imagem de um buraco negro encanta cientistas").

u. Explicações acerca da localização do buraco negro a que a imagem se refere.

v. Explicações acerca da possibilidade de que imagens futuras sejam melhores.

${ }^{8} \mathrm{O}$ vídeo se encontra na plataforma Youtube, disponível em https: //www.youtube.com/watch?v=FBchtofZJSM Acesso em: 12 abr. 2021.

9 O vídeo se encontra na plataforma Youtube, disponível en 10 Acesso em: 12 abr. 2021

10 JORNAL Nacional. Apresentado por Willian Bonner e Renata Vasconcellos. Rio de Janeiro: Rede Globo de Televisão, 11 abr. 2019, 20 h. Duração 2 minutos e 28 segundos. Reportagem acerca de como foram obtidas as primeiras imagens de um buraco negro. Disponível em: https://globoplay.globo.com/v/7534273/progra ma/ Acesso em: 12 abr. 2021. w. Representação figuras de buracos negros para ilustrar que algumas das imagens são concepções artísticas (Figura 3(a), (b) e (c)).

x. Simulação computacional acerca de buracos negros.

y. Solicitação, aos grupos de investigação, para que discutam o "questionário 3.4".

\subsubsection{Questionários e roteiros}

- Questionário 3.1 1) Elenque as diferenças entre o conceito de estrelas negras de John Michell e os buracos negros como definidos atualmente. 2) Explique por que, mesmo com uma solução exata das equações de Einstein, os cientistas da época não acreditavam na existência dos buracos negros. 3) Explique por que, somente após a segunda guerra mundial, o estudo dos buracos negros tomou fôlego.

- Questionário 3.2 1) Explique como o raio de Schwarzschild poderia ser utilizado para determinar o tamanho do buraco negro que um corpo celeste formaria se ele pudesse ser transformado em um. 2) Caso o Sol se tornasse um buraco negro, explique o que aconteceria com a órbita dos planetas no sistema solar. Qual seria o tamanho desse buraco negro? 3) Explique a relação entre o raio de Schwarzschild e o horizonte de eventos de um buraco negro.

- Questionário 3.3 1) Explique de que modo a pressão interna das estrelas é contrabalanceada pela gravidade. 2) Explique como pode ser feita a detecção de um buraco negro e elabore outro modo de detecção diferente dos mostrados nos exemplos dados.

- Questionário 3.4 1) De que modo os buracos negros representados nas figuras estão ligados à gravitação? 2) Por que se pode inferir que essas imagens representam buracos negros?

- Roteiro de discussão 3.1 1) Os cientistas antes de Einstein já tinham pensado em estruturas como os buracos negros? Como elas eram? Baseadas em que corrente científica? 2) Quem foram alguns dos cientistas que continuaram e complementaram os trabalhos de Einstein? Como foi esse processo? 3) Por que, inicialmente, os cientistas não acreditavam na existência real dos buracos negros, mesmo que os cálculos apontassem para sua existência? 4) Como a $2^{\text {a }}$ GM influenciou a ciência em geral? E a guerra fira?

- Roteiro de discussão 3.2 1) Como surgem os buracos negros? 2) O Sol poderia se transformar em um buraco negro? 3) Os buracos negros podem aumentar seu tamanho? E diminuir? 


\subsubsection{Análise das estratégias didáticas}

Nas fases de organização prévia, estratégias "a", "b", "h", "l" e "t", a maior parte dos estudantes foi capaz de reconhecer as Figuras 3a, 3p e 3t como sendo de buracos negros, revelando a existência de subsunçores acerca do tema em suas estruturas cognitivas. Além disso, durante a apresentação dos vídeos e da reportagem alguns estudantes demonstraram curiosidade científica acerca do tema e foram capazes de fazer inferências baseadas no conjunto de tópicos estudados, revelando habilidades de investigação e tradução.

Nas fases de investigação, estratégias "d", "e", "n", "o" e "q", as respostas de alguns estudantes se assemelharam às explicações presentes no texto de apoio e dadas pelo professor, revelando indícios de aprendizagem apenas mecânica, enquanto outros apresentaram respostas mais elaboradas e diferentes das apresentadas, demonstrando habilidades de raciocínio, de formação de conceitos, de investigação e de tradução, além de indícios da ocorrência de aprendizagem significativa dos tópicos estudados.

Nas fases de diferenciação progressiva, estratégias "c", "g", "i", "j", "r" e "u", os estudantes além de atentos, participaram com perguntas cuja qualidade revelou a utilização das habilidades de ordem superior de raciocínio, formação de conceitos, investigação e tradução.

Nas fases de avaliação de aprendizagem, estratégias "f", "k", "o" e "y", os estudantes apresentaram indícios de aprendizagem significativa acerca do tema buracos negros, bem como o uso de habilidades de ordem superior de raciocínio.

Nas fases de reconciliação integrativa, estratégias "s", "v", "w" e "x", os estudantes se mostraram entusiasmados com o tema e com a possibilidade da alteração de parâmetros durante a simulação. Os comentários, as perguntas e as sugestões para novos parâmetros de simulação, demonstram a utilização das habilidades de ordem superior de raciocínio, investigação, formação de conceitos e tradução, além de apontarem indícios de aprendizagem significativa acerca do tema buracos negros.

\subsection{Lentes gravitacionais}

\subsubsection{Estratégias didáticas}

a. Apresentação da figura "Cruz de Einstein", obtida pelo telescópio Hubble em 1990 (Figura 4).

b. Questionamentos, aos estudantes, acerca de quantos objetos diferentes eles observariam na figura.

c. Explicações acerca da existência de apenas dois objetos diferentes na figura: uma galáxia, ao centro, e quatro imagens do mesmo quasar ao redor dessa galáxia.

d. Explicações acerca da imagem que representa o fenômeno chamado lente gravitacional, previsto

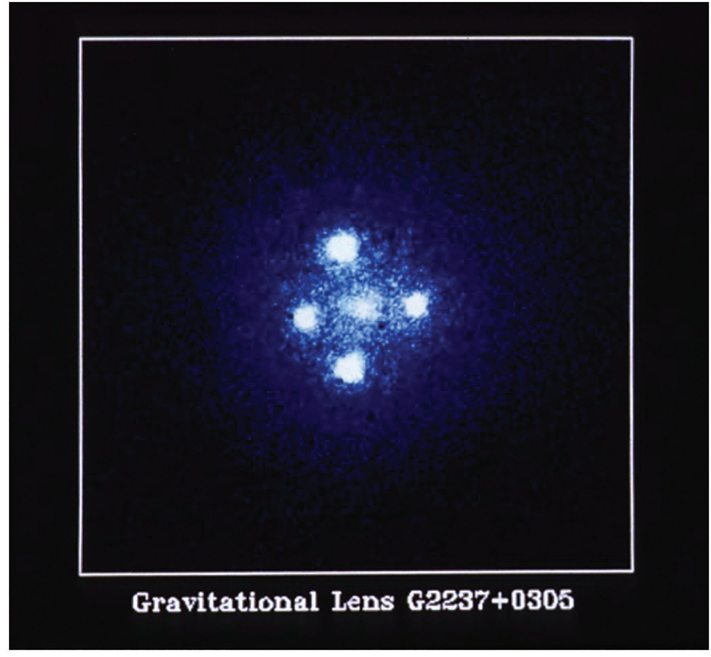

Figura 4: Lente gravitacional G2237+0305, também conhecida como Cruz de Einstein. Imagem obtida pelo telescópio Hubble em 1990

Fonte: ESA/Hubble, 1990 [24].

pela Relatividade Geral como decorrente da curvatura do espaço-tempo provocada por corpos massivos.

e. Formação de pequenos grupos de investigação para discutir o "texto de apoio 4.1".

f. Explicações acerca das lentes gravitacionais com o uso de imagens projetadas.

g. Solicitação, aos grupos de investigação, para que discutam os itens do "questionário 4.1".

h. Solicitação, aos grupos de investigação, para que produzam vídeo (de até dois minutos) para explicar o que seriam as lentes gravitacionais e sua importância para a astronomia e cosmologia.

i. Simulação computacional acerca de lentes gravitacionais produzidas por buracos negros.

j. Solicitação, aos grupos de investigação, para que discutam o "texto de apoio 4.2".

k. Explicações acerca das lentes gravitacionais, com análise de imagem produzidas nas simulações.

1. Solicitação, aos grupos de investigação, para que discutam o "questionário 4.2".

\subsubsection{Questionários e figuras}

Questionário 4.1 1) Observe a Figura 5(a), para, então, responder às questões apresentadas. a) Porque há objetos deformados em meio a objetos não deformados na Figura? b) Onde está localizada a maior concentração de massa do aglomerado? Explique sua resposta. 2) Na Figura 5(b), pode-se observar duas galáxias atuando para a formação do fenômeno conhecido como Anel de Einstein. a) Explique o papel de cada galáxia na formação do Anel de Einstein da Figura 5b. 4) Um buraco 


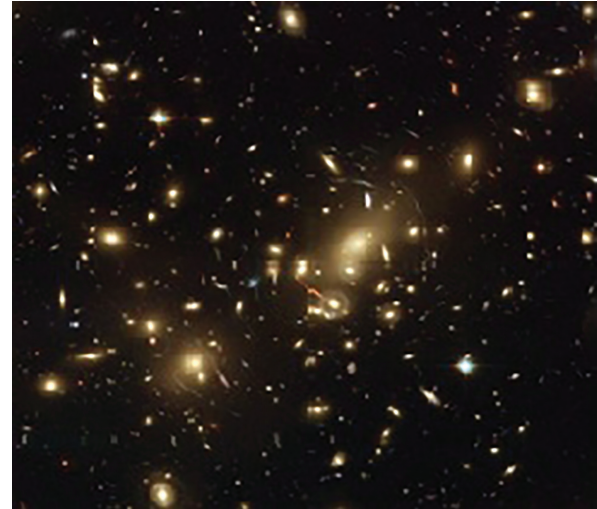

(a)

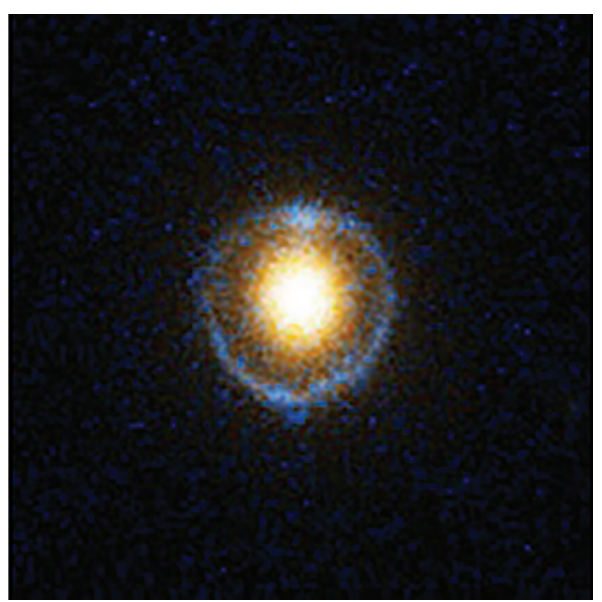

(b)

Figura 5: Imagens do telescópio Hubble: (a) Aglomerado de galáxias Abell 2218 (2008) [25]; e (b) Anel de Einstein (2005) [26].

negro pode atuar como uma lente gravitacional? Explique sua resposta.

- Questionário 4.2 1) Explique como as lentes gravitacionais poderiam ser utilizadas para entender como algumas estruturas do universo evoluem. 2) Explique como uma lente gravitacional poderia ser utilizada na visualização de uma estrela e de um exoplaneta no espaço.

\subsubsection{Análise das estratégias didáticas}

Nas fases de organização prévia, estratégias "a", "b" e "c", ao observar a figura "cruz de Einstein", alguns estudantes entenderam haver apenas um objeto, uma estrela, replicado quatro vezes, enquanto outros diziam haver cinco objetos diferentes, que seriam galáxias. As expressões destes entendimentos indicaram a presença de subsunçores acerca da astronomia básica, mas também a ausência de outros relacionados às consequências da deformação espaço-temporal necessários para o desenvolvimento do módulo de lentes gravitacionais.

Nas fases de investigação, estratégias "e" e "j", os estudantes ficaram atentos e interessados em saber a que corresponderiam cada uma das imagens apresentadas. As manifestações, por meio de perguntas, revelaram interesse pelo tema lentes gravitacionais e desenvolvimento das habilidades de raciocínio, formação de conceitos, investigação e tradução.

As fases de diferenciação progressiva, estratégias "d", "f" e "k", chamaram a atenção dos estudantes, principalmente quando as imagens eram projetadas na parede. Ao serem questionados acerca de onde estaria a maior concentração de massa em cada uma das imagens projetadas, alguns alunos conseguiram identificá-las corretamente, demonstrando indícios de aprendizagem significativa acerca do tema.

Nas fases de avaliação de aprendizagem, estratégias "g", "h" e "l", chamou a atenção o fato de nenhum dos estudantes ter realizado a tarefa. Quando questionados acerca dos motivos, informaram não terem tido vontade de produzir o vídeo. O professor relata que a não realização de atividades extra-classe é usual entre o grupo de estudantes analisado, mesmo quando fornecidas orientações e prazo adequado. Esse aspecto, em particular, corresponde a importante objeto de investigações complementares a esta.

Na fase de reconciliação integrativa, estratégia "i", os estudantes se mostraram entusiasmados e conseguiram identificar os tipos de lentes gravitacionais formadas, demonstrando indícios de aprendizagem significativa. Além disso, os estudantes ainda expressaram curiosidade acerca do fenômeno e o professor utilizou esse sentimento para fazer uma breve revisão dos tópicos passados, fazendo sua conexão com as lentes gravitacionais (Figura 5].

\section{Análise dos Questionários}

Uma das estratégias presentes em todos os módulos didáticos foi a utilização de questionários como instrumento auxiliar para inferências acerca da ocorrência de aprendizagem significativa. Foram aplicados onze questionários, com um total de trinta e seis questões, acerca dos temas abordados nas aulas. Os questionários foram respondidos em grupos pelos estudantes e, considerando as ausências às aulas, um total de 243 respostas foram analisadas e classificadas em cinco níveis, conforme o grau de conhecimento demonstrado:

N1. Questões deixadas em branco.

N2. Respostas em desacordo com a matéria de ensino. Os estudantes não demonstram qualquer indício de aprendizagem significativa acerca do tópico abordado.

N3. Respostas que demonstram entendimento precário da matéria de ensino. Os estudantes demonstram indícios de aprendizagem mecânica ou de confusão entre os conceitos abordados, mas não há indícios de aprendizagem significativa.

N4. Respostas que demonstram entendimento intermediário da matéria de ensino. Os estudantes 
Tabela 1: Número de respostas avaliadas, em cada nível, para cada questionário.

\begin{tabular}{|c|c|c|c|c|c|c|c|c|}
\hline \multicolumn{3}{|c|}{ NÍVEIS } & N5 & $\mathrm{N} 4$ & N3 & N2 & N1 & TOTAL \\
\hline \multirow{10}{*}{ 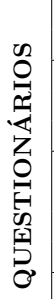 } & \multirow{2}{*}{ M1 } & Q1 & 3 & 11 & 5 & 2 & 0 & 21 \\
\hline & & Q2 & 0 & 9 & 4 & 2 & 0 & 15 \\
\hline & \multirow{3}{*}{ M2 } & Q3 & 7 & 8 & 12 & 9 & 4 & 40 \\
\hline & & $\mathrm{Q} 4$ & 1 & 6 & 4 & 11 & 10 & 32 \\
\hline & & Q5 & 0 & 11 & 11 & 10 & 0 & 32 \\
\hline & \multirow{4}{*}{ M3 } & Q6 & 0 & 4 & 9 & 5 & 0 & 18 \\
\hline & & Q7 & 0 & 4 & 5 & 4 & 2 & 15 \\
\hline & & $\mathrm{Q} 8$ & 1 & 1 & 11 & 1 & 0 & 14 \\
\hline & & Q9 & 1 & 4 & 3 & 3 & 1 & 12 \\
\hline & \multirow{2}{*}{ M4 } & Q10 & 1 & 8 & 12 & 3 & 6 & 30 \\
\hline & & Q11 & 0 & 2 & 8 & 4 & 0 & 14 \\
\hline \multicolumn{3}{|c|}{ TOTAL } & 14 & 68 & 84 & 54 & 23 & 243 \\
\hline & & & \multicolumn{2}{|c|}{$33.7 \%$} & $34,6 \%$ & \multicolumn{2}{|c|}{$31,7 \%$} & \\
\hline & & & \multicolumn{2}{|c|}{ significativa } & mecânica & \multicolumn{2}{|c|}{$\begin{array}{c}\text { respostas } \\
\text { em branco/ } \\
\text { desconexas }\end{array}$} & \\
\hline & & & \multicolumn{5}{|c|}{ Tipo de aprendizagem } & \\
\hline
\end{tabular}

demonstram indícios de aprendizagem significativa ao expor entendimento das relações entre os fenômenos, porém ainda não conseguem expressálas de forma completa.

N5. Respostas que demonstram entendimento adequado da matéria de ensino. Os estudantes demonstram indícios de aprendizagem significativa e conseguem expor suas ideias de forma adequada.

A Tabela 1 apresenta a distribuição das respostas aos itens dos questionários de acordo com os níveis, conforme o grau de conhecimento demonstrado. Os dados revelam indícios de aprendizagem significativa em 33,7\% das respostas avaliadas e indícios de aprendizagem mecânica em $34,6 \%$ delas, indicando que o produto educacional atendeu parcialmente a seus objetivos. Em relação à parte insatisfatória dos resultados, cabe mencionar que o conteúdo ministrado representou novidade aos estudantes e, mesmo tendo sido transposto para o ensino médio, possui grau de complexidade acima daquele a que são comumente submetidos em seus cotidianos escolares, impactando em sua assimilação. Segundo Ausubel \& Robinson [15] e Ausubel [15], a aprendizagem significativa é prejudicada quando há ausência ou baixa qualidade dos subsunçores na estrutura cognitiva dos estudantes. Em Ferreira et al. [1], é possível encontrar uma discussão apropriada e atualizada acerca do levantamento de conhecimentos prévios e, quando aplicável, a realização de organização avançada de subsunçores.

Também é preciso considerar o efeito das ausências dos estudantes às aulas. A estrutura do produto educacional foi montada de modo que os conteúdos dos módulos anteriores servissem de subsunçores para os módulos posteriores. Elas comprometeram a ministração de parte do conteúdo para o grupo de estudantes analisado e tornou difícil a formação, estabilização e modificação de seus subsunçores, impactando a aprendizagem significativa da matéria de ensino.

Desse modo, é interessante incluir como indicador de sucesso do produto educacional a aprendizagem mecânica dos tópicos de ensino, pois os estudantes pas- saram do estado em que possuíam poucos subsunçores acerca dos conteúdos para outro estado em que a aprendizagem, mesmo tendo sido assimilada de forma arbitrária ou literal pela estrutura cognitiva, poderia servir de base para a aprendizagem significativa desses tópicos, caso houvesse engajamento dos estudantes em uma revisão ou mesmo em complementar os assuntos abordados.

\section{Considerações Finais}

A teoria de gravitação de Newton e teoria da relatividade geral de Einstein foram combinadas para a construção de quatro módulos de ensino envolvendo gravitação e sistema solar, relatividade geral, buracos negros, e lentes gravitacionais. As aulas apresentaram atividades diversificadas e a utilização de tecnologias educacionais (vídeos, demonstrações, simulações etc.), despertando o entusiasmo e o interesse dos estudantes. A formação de comunidades de investigação permitiu que os estudantes se organizassem e se ajudassem durante realização das diversas estratégicas didáticas (discussão dos textos de apoio, análise dos vídeos, imagens, simulações etc.).

Os relatos do professor (e dos estudantes) revelaram que o entusiasmo e interesse dos estudantes pelos temas tratados foi maior que o demonstrado nas aulas regulares, principalmente durante as atividades que envolviam simulações ou demonstrações.

As comunidades de investigação estiveram ativas durante as aulas, com estudantes participativos nas discussões e com perguntas ou comentários adicionais ao professor e à turma acerca dos temas estudados. A partir da qualidade das respostas aos questionamentos e dos comentários realizados pelos estudantes, com abordagens diferentes das oferecidas nos textos de apoio e nas explicações do professor, foi possível perceber que as habilidades cognitivas de ordem superior dos estudantes foram estimuladas, permitindo inferir que o pensar superior também se desenvolveu.

Indícios de ocorrência de aprendizagem significativa foram verificados a partir da qualidade da argumentação dos estudantes durante as discussões e pelo nível adequado de respostas acerca de um terço das perguntas dos questionários respondidos. É importante registar que, no início da intervenção pedagógica, os estudantes possuíam nenhum ou poucos subsunçores adequados aos temas propostos, e neste caso, embora o último terço tenha mostrado respostas desconexas ou nenhuma resposta, não indicando a ocorrência de qualquer tipo de aprendizagem significativa ou mecânica, o resultado, de modo geral, se revela parcialmente satisfatório, pois demonstra a evolução de seus conceitos.

A verificação da ausência ou da baixa qualidade dos subsunçores dos estudantes relativos a alguns temas sugere a possibilidade de aprimoramento do produto educacional pela adição de um módulo anterior. Neste caso, temas como história da ciência e da gravitação, 
filosofia natural de Aristóteles, e a quebra de paradigma defendida por Galileu, poderiam minimizar esses problemas. O módulo extra poderia subsumir estruturas cognitivas dos estudantes, viabilizando a aprendizagem significativa dos tópicos estudados durante a realização das várias estratégias didáticas. Além disso, os conteúdos desse novo módulo poderiam estimular discussões de qualidade dentro das comunidades de investigação e, como consequência, auxiliar o desenvolvimento do pensamento de ordem superior.

Em síntese, a articulação teórico-metodológica fundamentada em Lipman e Ausubel, para desenvolvimento de produto educacional acerca de tópicos de astronomia para o ensino médio, revelou potencial para estimular as habilidades de ordem superior dos estudantes e, ao mesmo tempo, promover aprendizagem significava. Também sugeriu uma possibilidade para minimizar a precariedade do ensino de astronomia em condições típicas da educação pública, fornecendo aos estudantes uma alternativa de aprendizagem não limitada a um currículo desgastado e baseado em propostas metodológicas difusas.

\section{Agradecimentos}

Ao Prof. Ivan Soares Ferreira (in memorian), pela leitura e por contribuições iniciais à concepção teórica e ao produto educacional de que deriva este artigo; e ao Licenciado Palton Alves Lima, pelo suporte a diversas ações de pesquisa do PPG em Ensino de Física da UnB.

\section{Referências}

[1] M. Ferreira, O.L. Silva Filho, M.A. Moreira, G.B. Franz, K.O. Portugal e D.X.P. Nogueira, Rev. Bras. Ensino Fís. 42, e20200057 (2020).

[2] A.L.D. Froes, Rev. Bras. Ensino Fís. 36, 1 (2014).

[3] http://portal.mec.gov.br/seb/arquivos/pdf/Ciencia sNatureza.pdf acessado 11/03/2020.

[4] http://basenacionalcomum.mec.gov.br/images/hist orico/BNCC_EnsinoMedio_embaixa_site_110518.pd f. acessado 11/03/2020.

[5] R.V.L. do Couto, Astronomia no Ensino Médio: uma abordagem simplificada a partir da Teoria da Relatividade Geral. Dissertação de Mestrado, Universidade de Brasília, Brasília (2020).

[6] E.C. Junior, B.S. Fernandes, G.S. Lima, A.J. Siqueira, J.N.M. Paiva, M.G. Santos, J.P. Tavares, T.V. Souza e T.M.F. Gomes, Rev. Bras. Ensino Fís. 40, e5401 (2018).

[7] M.R.R. Sá, Teoria da Relatividade Restrita e Geral ao longo do $1^{o}$ ano do ensino médio: uma proposta de inserção. Dissertação de Mestrado, Universidade de Brasília, Brasília (2015).

[8] J.C. Freire, Evolução de conceitos de mundo: uma proposta para inserção da teoria da relatividade no ensino médio. Dissertação de Mestrado, Universidade Federal de Lavras, Lavras (2015).
[9] H.F.F. Souza, A cosmologia de Newton a Einstein: uma proposta para o ensino médio com recursos de hipermídia. Dissertação de Mestrado, Universidade Federal do Rio de Janeiro, Macaé (2017).

[10] V.H.R. Oliveira, Alguns aspectos da física de buracos negros através da modelagem matemática: uma intervenção didática para o ensino médio. Dissertação de Mestrado, Instituto Federal de Educação, Ciência e Tecnologia Fluminense, Goytacazes (2017).

[11] R.V. Do Couto, M. Ferreira e O.L. Silva Filho, Revista do Professor de Física 3, 15 (2019).

[12] O.L. Silva Filho, M. Ferreira, A.M.M. Polito e A.L.M.B. Coelho, Pesquisa e Debate em Educação 11, 1 (2020).

[13] O.L. Silva Filho e M. Ferreira, Revista do Professor de Física 2, 104 (2018).

[14] D. Ausubel e F.G. Robinson, School Learning: An Introduction to Educational Psychology (Holt, Rinehart and Winston, Michigan, 1969).

[15] D. Ausubel, Educational Psychology: A Cognitive View (Holt, Rinehart and Winston, Michigan, 1968).

[16] M.A. Moreira, Teorias de Aprendizagem (EPU, São Paulo, 2011).

[17] R.M. Pontili e A.L. Kassouf, Revista de Economia e Sociologia Rural 45, 27 (2007).

[18] M.R. Costa, E.S. Guimarães e S.M.O. Rocha, Ensino \& Multidisciplinaridade 1, 122 (2015).

[19] CODEPLAN e SEPLAG, Pesquisa distrital por amostra de domicícios PDAD - 2015 - Recanto das Emas (CODEPLAN, Brasília, 2015).

[20] G. Army, Jogo eletrônico Universe Sandbox, disponível em: https://store.steampowered.com/app /230290, acessado 02/06/2020.

[21] NASA, Black Hole Art, disponível em: https://ww w.nasa.gov/sites/default/files/images/729665ma in_A-BlackHoleArt-pia16695_full.jpg, acessado 02/06/2020.

[22] NASA, Fast-flying Black Hole, disponível em: https: //www.nasa.gov/sites/default/files/thumbnails/im age/hs-2002-30-a-full_jpg.jpg, acessado 02/06/2020.

[23] EHT, Astronomers Capture First Image of a Black Hole, disponível em: https://eventhorizontelescope. org/press-release-april-10-2019-astronomers-captur e-first-image-black-hole\#, acessado 02/06/2020.

[24] ESA/HUBBLE, Gravitational Lens G2237+0305, disponível em: https://imgsrc.hubblesite.org/hvi/up loads/image_file/image_attachment/159/full_tif.tif acessado 02/06/2020.

[25] ESA/Hubble, Abell 2218, disponível em: https://ww w.spacetelescope.org/images/heic0814a/, acessado $02 / 06 / 2020$.

[26] ESA/Hubble, Einstein Ring, disponível em: https: //www.spacetelescope.org/static/archives/images/ original/opo0532g.tif acessado 02/06/2020. 